\title{
Limit Cycle Generation for Multi-Modal and 2-Dimensional Piecewise Affine Control Systems
}

\author{
Tatsuya Kai \\ Department of Applied Electronics \\ Faculty of Industrial Science and Technology \\ Tokyo University of Science \\ 6-3-1 Niijuku, Katsushika-ku, Tokyo 125-8585 JAPAN \\ Email: kai@rs.tus.ac.jp
}

\begin{abstract}
This paper considers a limit cycle control problem of a multi-modal and 2-dimensional piecewise affine control system. Limit cycle control means a controller design method to generate a limit cycle for given piecewise affine control systems. First, we deal with a limit cycle synthesis problem and derive a new solution of the problem. In addition, theoretical analysis on the rotational direction and the period of a limit cycle is shown. Next, the limit cycle control problem for piecewise affine control system is formulated. Then, we obtain matching conditions such that the piecewise affine control system with the state feedback law corresponds to the reference system which generates a desired limit cycle. Finally, in order to indicate the effectiveness of the new method, a numerical simulation is illustrated.
\end{abstract}

\section{INTRODUCTION}

Limit cycles are known to be quite important concept in various research fields [1]. We can find limit cycles in real world, for example, stable walking or gaits of humanoid robots in robotic engineering, oscillator circuits in electronic engineering, catalytic hypercycles in chemistry, circadian rhythms in biology, boom-bust cycles in economics and so on. Researches on limit cycles have been eagerly done from both mathematical and engineering perspectives so far [2], [3], [4], [5], [6], [7], [8], [9], [11], [12], [13], [14]. Especially, some conditions for nonlinear systems that generate periodic solutions and some applications were shown in [2], and in [7], a synthesis method of hybrid systems whose solution trajectories converge to desired trajectories was proposed. In these studies, it is guaranteed that solution trajectories of the systems converges to a desired closed curve, and the existence of limit cycles was confirmed by numerical simulations, However, the mathematical guarantee of the existence of limit cycles was not shown. On the other hand, the authors proposed a synthesis method of multi-modal and 2-dimensional piecewise affine systems that generate desired limit cycles in [10], [15] and showed a mathematical proof of the existence and the uniqueness of a limit cycle for the proposed system. In addition, some theoretical analysis on the rotational direction and the period of a limit cycle is derived. In this study, we assume that the whole of a system can be designed. A method to generate a desired limit cycle for a given piecewise affine control system with tuning some parameters of the system is more useful for a wide variety of situations. However, such a control method have not been proposed so far.

Hence, we consider a limit cycle control problem of multimodal and 2-dimensional piecewise affine systems in this paper. The outline of this paper is as follows. We first consider a limit cycle synthesis problem and derive its new solution in Section II. Some theoretical properties are also shown. Then, in Section III, a formulation of limit cycle control problem is presented, and necessary and sufficient conditions for the problem, which are called matching conditions, are derived. Finally, a numerical simulation is shown in order to confirm the effectiveness of the new method in Section IV.

\section{LIMIT CYCLE SYNTHESIS OF PIECEWISE AFFINE SYSTEMS}

\section{A. Formulation of Limit Cycle Synthesis}

In this section, we consider a synthesis problem of piecewise affine systems which generate desired limit cycles. First, this subsection give the formulation of the problem. Consider the 2-dimensional Euclidian space: $\mathbf{R}^{2}$, its coordinate: $x=\left[\begin{array}{ll}x_{1} & x_{2}\end{array}\right]^{\top} \in \mathbf{R}^{2}$, and the origin of $\mathbf{R}^{2}: O$. Let us set $N(N \geq 3)$ points $P_{i} \neq O(i=1, \cdots, N)$ in $\mathbf{R}^{2}$ and denote the vector from $O$ to $P_{i}$ by $p_{i}=\left[\begin{array}{ll}p_{i}^{1} & p_{i}^{2}\end{array}\right]^{\top}$. We also denote the angle between the half line $O P_{i}$ and the $x_{1}$-axis by $\theta_{i}$. Now, without loss of generality, we assume that the $N$ points $P_{i}(i=1, \cdots, N)$ are located in the counterclockwise rotation from the $x_{1}$-axis, that is, $0 \leq \theta_{1}<\theta_{2}<\cdots<\theta_{N}$ holds.

Next, we define the semi-infinite region $D_{i}$ which is sandwiched by the half lines $O P_{i}$ and $O P_{i+1}$ and the line segment $C_{i}$ joining $P_{i}$ and $P_{i+1}$, where $P_{N+1}=P_{1}$. Set a polygon that is a union of $C_{i}(i=1, \cdots, N)$ as

$$
C:=\bigcup_{i=1}^{N} C_{i} .
$$

Fig. 1 shows an example of a Polygonal Closed Curve for $N=5$.

We then consider the affine system defined in $D_{i}$ :

$$
\dot{x}=a_{i}+A_{i} x, \quad x \in D_{i}
$$

where $x=\left[\begin{array}{ll}x_{1} & x_{2}\end{array}\right]^{\top} \in \mathbf{R}^{2}$ is the state variable, and $a_{i} \in \mathbf{R}^{2}, A_{i}=\mathbf{R}^{2 \times 2}$ are the affine term and the coefficient matrix, respectively. Consequently, we treat the $N$-modal and 2-dimensional piecewise affine system that consists of $N$ regions $D_{i}(i=1, \cdots, N)$ and $N$ affine systems (2). In this paper, we consider the following synthesis problem on limit cycles called "the limit cycle synthesis problem." 


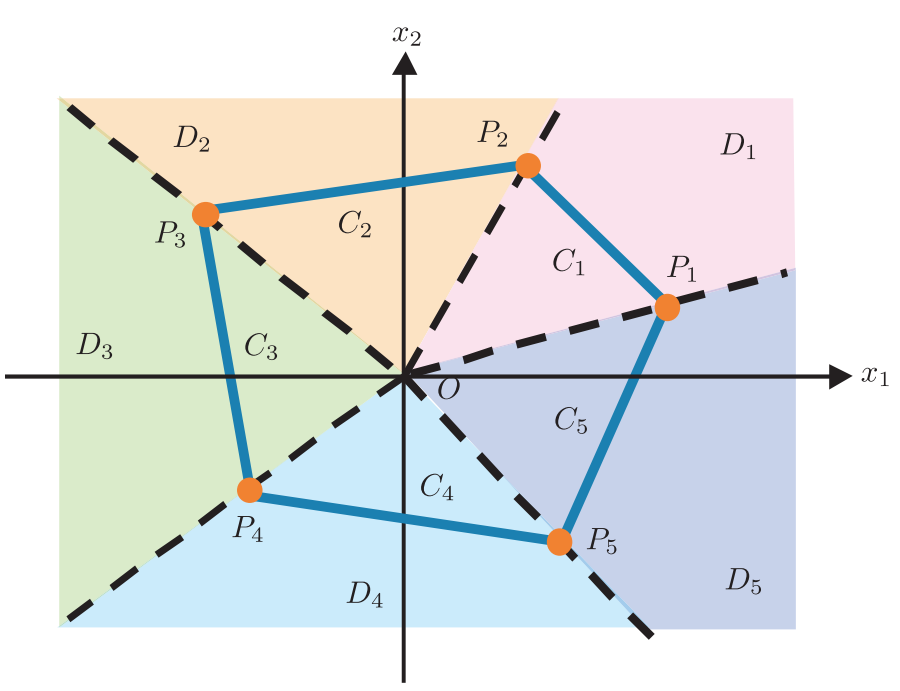

Fig. 1 : Example of Polygonal Closed Curve $(N=5)$

Problem 1 : For the $N$-modal and 2-dimensional piecewise affine system (2), design $a_{i}, A_{i}(i=1, \cdots, N)$ such that a given polygonal closed curve $C(1)$ is a unique and stable limit cycle pf the system.

A solution of Problem 1 has been derived in the author's previous studies [10], [15]. However, in this paper we will derive another solution which can be utilized to consider the limit cycle control problem shown in Section III.

\section{B. Proposed System and Existence/Uniqueness of Limit Cycle}

Next, we shall derive a solution for Problem 1 in this subsection. We also consider the existence and the uniqueness of a limit cycle for the obtained system. We focus on a behavior of a solution trajectory of (2) in $D_{i}$. It is easily confirmed that the equation of $C_{i}$ is represented by

$$
\begin{aligned}
\left(p_{i}^{2}-p_{i+1}^{2}\right) x_{1} & -\left(p_{i}^{1}-p_{i+1}^{1}\right) x_{2} \\
& +p_{i}^{1} p_{i+1}^{2}-p_{i}^{2} p_{i+1}^{1}=0 .
\end{aligned}
$$

Using (3), we now define a limit cycle function $V_{i}$ as

$$
\begin{aligned}
V_{i}(x)=\left(p_{i}^{2}-p_{i+1}^{2}\right) & x_{1}-\left(p_{i}^{1}-p_{i+1}^{1}\right) x_{2} \\
& +p_{i}^{1} p_{i+1}^{2}-p_{i}^{2} p_{i+1}^{1} .
\end{aligned}
$$

If $V_{i}$ converges to 0 along a solution trajectory of (2), then the solution trajectory of (2) also converges to $C_{i}$. Hence, $a_{i}$ and $A_{i}$ should be determined so that $V_{i}$ converges to 0 along a solution trajectory of (2). Now, an important result on design of nonlinear systems is shown as follows [2].

Theorem 1 [2] : Consider the 2-dimensional nonlinear system:

$$
\dot{x}=f(x)+g(x) \text {, }
$$

where $x \in \mathbf{R}^{2}$ and $f, g \in \mathbf{R}^{2} \rightarrow \mathbf{R}^{2}$ are vector fields defined in $\mathbf{R}^{2}$. In addition, consider a radial and unbounded function define on $\mathbf{R}^{2}: V: \mathbf{R}^{2} \rightarrow \mathbf{R}$ such that $V(0)=0$ and $V(x) \neq$ $0, \forall x \neq 0$ hold. We now define $f$ and $g$ as

$$
f:=U_{f}(x) \frac{\partial V^{\top}}{\partial x}, g:=-u_{g}(V) U_{g}(x) \frac{\partial V^{\top}}{\partial x},
$$

where a skew-symmetric matrix $U_{f}$, a positive definite matrix $U_{g}$, and $u_{g}$ such that $u_{g}(V)>0, V \neq 0$ holds. Then, for the system (5) with (6),

$$
\lim _{t \rightarrow \infty} V(x(t))=0
$$

holds

Applying Theorem 1, we can derive an affine term $a_{i}$ and a coefficient matrix $A_{i}$ of the affine system (2) such that $V_{i}$ converges to 0 along a solution trajectory of (2). As a specific form of (5) and (6), we use

$$
\begin{aligned}
\dot{x} & =f_{i}+g_{i}, \\
f_{i} & :=\left[\begin{array}{cc}
0 & \omega_{i} \\
-\omega_{i} & 0
\end{array}\right] \frac{\partial V_{i}^{\top}}{\partial x}, \\
g_{i} & :=-V_{i}(x)\left[\begin{array}{cc}
\lambda_{i} & 0 \\
0 & \lambda_{i}
\end{array}\right] \frac{\partial V_{i}^{\top}}{\partial x},
\end{aligned}
$$

where $\omega_{i}$ and $\lambda_{i}>0$ are design parameters. Substituting (4) into (8) and comparing it with (2), we can obtain $a_{i}$ and $A_{i}$ of (2) as

$$
\begin{aligned}
& a_{i}= \\
& {\left[\begin{array}{c}
-\lambda_{i}\left(p_{i}^{2}-p_{i+1}^{2}\right)\left(p_{i}^{1} p_{i+1}^{2}-p_{i}^{2} p_{i+1}^{1}\right)-\omega_{i}\left(p_{i}^{1}-p_{i+1}^{1}\right) \\
\lambda_{i}\left(p_{i}^{1}-p_{i+1}^{1}\right)\left(p_{i}^{1} p_{i+1}^{2}-p_{i}^{2} p_{i+1}^{1}\right)-\omega_{i}\left(p_{i}^{2}-p_{i+1}^{2}\right)
\end{array}\right],} \\
& A_{i}= \\
& {\left[\begin{array}{cc}
-\lambda_{i}\left(p_{i}^{2}-p_{i+1}^{2}\right)^{2} & \lambda_{i}\left(p_{i}^{2}-p_{i+1}^{2}\right)\left(p_{i}^{1}-p_{i+1}^{1}\right) \\
\lambda_{i}\left(p_{i}^{2}-p_{i+1}^{2}\right)\left(p_{i}^{1}-p_{i+1}^{1}\right) & -\lambda_{i}\left(p_{i}^{1}-p_{i+1}^{1}\right)^{2}
\end{array}\right] .}
\end{aligned}
$$

Compared to the piecewise affine system shown in [10], [15], (28) contains a new parameter $\lambda_{i}$ and this additional parameter plays an important role in the limit cycle control problem in Section III. It is noted that that the system (2) with (28) satisfies only the convergence property (7), that is, its solution trajectory converges to $C_{i}$ in $D_{i}$. Hence, we will discuss the existence of a unique and stable limit cycle of the system (2) with (28). To prove this, we first indicate three lemmas, and then we show the main theorem by using them. Now, we give the definition on the clockwise and counterclockwise rotations of limit cycle solution trajectories of the system (2) with (28) [10], [15].

Definition 1 [10], [15] : For limit cycle solution trajectories of the $N$-modal and 2-dimensional piecewise affine system (2) with (28), one that rotates in the clockwise direction in $\mathbf{R}^{2}$ is called a limit cycle solution trajectory in the clockwise rotation. On the contrary, one that rotates in the counterclockwise direction in $\mathbf{R}^{2}$ is called a limit cycle solution trajectory in the counterclockwise rotation (see Fig. 2).

Let us define a subset in $D_{i}$ as

$$
M_{i}\left(\varepsilon_{i}\right):=\left\{x \in D_{i} \mid \varepsilon_{i}^{-} \leq V_{i}(x) \leq \varepsilon_{i}^{+}\right\},
$$

where $\varepsilon_{i}^{-}, \varepsilon_{i}^{+} \in \mathbf{R}$ satisfies $\varepsilon_{i}^{-}<\varepsilon_{i}^{+}$and we set $\varepsilon_{i}=\left(\varepsilon_{i}^{-}, \varepsilon_{i}^{+}\right)$. We also define a sum of these subsets as

$$
M(\varepsilon):=\bigcup_{i=1}^{N} M_{i}\left(\varepsilon_{i}\right),
$$

where we use the notations: $\varepsilon^{-}=\left(\varepsilon_{1}^{-}, \cdots, \varepsilon_{N}^{-}\right), \varepsilon^{+}=$ $\left(\varepsilon_{1}^{+}, \cdots, \varepsilon_{N}^{+}\right), \varepsilon=\left(\varepsilon^{-}, \varepsilon^{+}\right)$, and the parameters $\varepsilon^{-}$and $\varepsilon^{+}$ 
are determined such that

$$
\begin{aligned}
& M^{-}\left(\varepsilon^{-}\right)=\bigcup_{i=1}^{N}\left\{x \in D_{i} \mid V_{i}(x)=\varepsilon_{i}^{-}\right\} \\
& M^{+}\left(\varepsilon^{+}\right)=\bigcup_{i=1}^{N}\left\{x \in D_{i} \mid V_{i}(x)=\varepsilon_{i}^{+}\right\}
\end{aligned}
$$

form closed polygons. If $M^{-}\left(\varepsilon^{-}\right)$and $M^{+}\left(\varepsilon^{+}\right)$are closed polygons, that is, $M(\varepsilon)$ is a bounded and closed set, then $\varepsilon$ is said to be admissible (see Fig. 3). We can derive the following proposition on $M(\varepsilon)$.

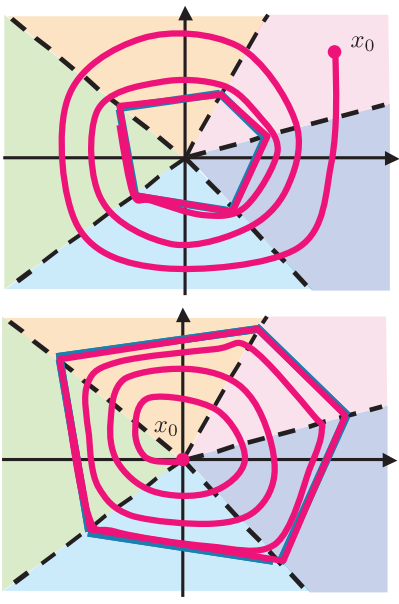

[a] Crockwise Rotation
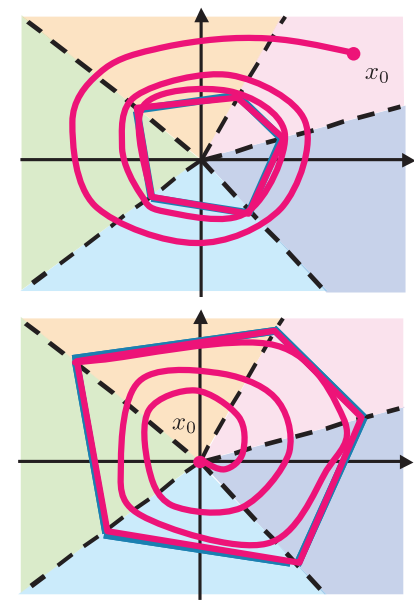

[b] Countercrockwise Rotation
Fig. 2 : Clockwise and Counterclockwise Rotations of Limit Cycle Solution Trajectories

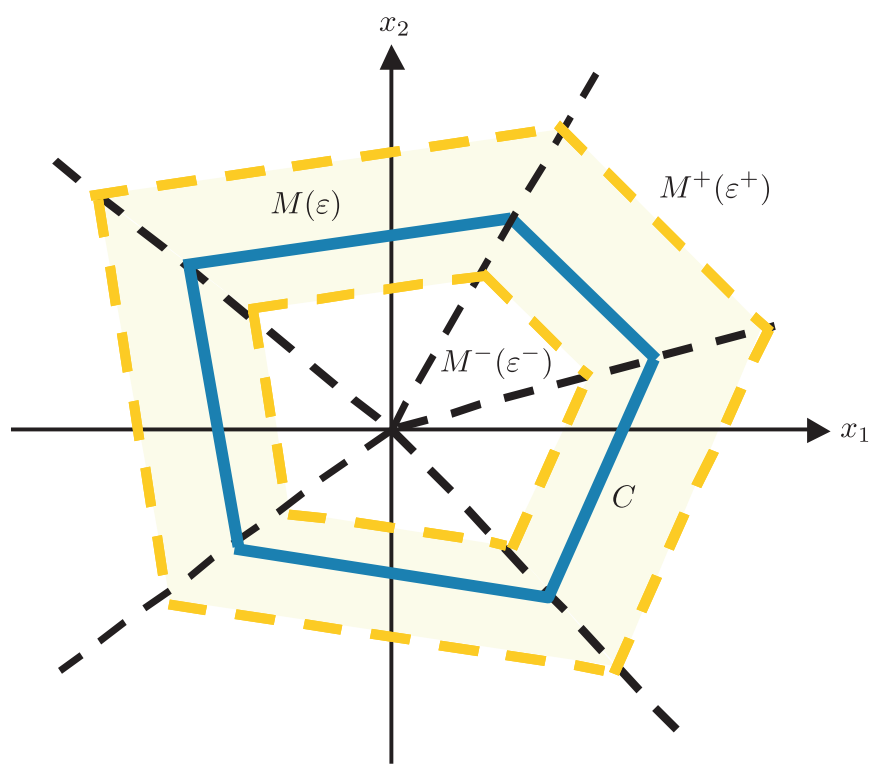

Fig. 3 : Example of $M^{-}\left(\varepsilon^{-}\right), \quad M^{+}\left(\varepsilon^{+}\right)$and $M(\varepsilon)$

Lemma 1 : For the $N$-modal and 2-dimensional piecewise affine system (2) with (28), $M(\varepsilon)$ is a positively invariant, bounded and closed set for any admissible $\varepsilon$.

(Proof) Calculating the time differential of $1 / 2 V_{i}^{2}$ along a solution trajectory of the system (2) with (28), we have

$$
\begin{aligned}
\frac{1}{2} \frac{d}{d t}\left(V_{i}^{2}\right)= & V_{i} \dot{V}_{i}=V_{i} \frac{\partial V_{i}}{\partial x} \dot{x}=V_{i} \frac{\partial V_{i}}{\partial x}\left(f_{i}+g_{i}\right) \\
= & V_{i} \frac{\partial V_{i}}{\partial x}\left[\begin{array}{cc}
0 & \omega_{i} \\
-\omega_{i} & 0
\end{array}\right] \frac{\partial V_{i}^{\top}}{\partial x} \\
& \quad-V_{i}^{2} \frac{\partial V_{i}}{\partial x}\left[\begin{array}{cc}
\lambda_{i} & 0 \\
0 & \lambda_{i}
\end{array}\right] \frac{\partial V_{i}^{\top}}{\partial x} \\
= & -\lambda_{i}^{2} V_{i}^{2} \frac{\partial V_{i}}{\partial x} \frac{\partial V_{i}^{\top}}{\partial x}<0 .
\end{aligned}
$$

Hence, it turns out that the velocity vector field of the system (2) with (28) points to the direction of the inner side of the bounded and closed set $M$ at any points on the boundary $M^{-}\left(\varepsilon^{-}\right) \cup M^{+}\left(\varepsilon^{+}\right)$of $M$. Consequently, $M(\varepsilon)$ is a positively invariant, bounded and closed set.

Next, we consider equilibrium points of the system (2) with (28). The following lemma on equilibrium points can be obtained.

Lemma 2 : Assume $\omega_{i} \neq 0(i=1, \cdots, N)$. Then, the $N$-modal and 2-dimensional piecewise affine system (2) with (28) does not have any equilibrium points in $M(\varepsilon)$ for any admissible $\varepsilon$.

(Proof) The unit vector which is on a parallel with $C_{i}$ in $D_{i}$ and points to the counterclockwise rotation is given by $\left(p_{i}-p_{i+1}\right) /\left\|p_{i}-p_{i+1}\right\|$. By considering the inner product of this unit vector and the velocity vector field of the system (2) with (28), we have the magnitude of the velocity component to the direction of $p_{i}-p_{i+1}$ for a solution trajectory $v_{i}$ of the system (2) with (28) in $D_{i}$ as

$$
v_{i}=\left(a_{i}+A_{i} x\right) \cdot \frac{p_{i}-p_{i+1}}{\left\|p_{i}-p_{i+1}\right\|} .
$$

Now, we denote a point in $D_{i}$ by $x=\alpha_{i} p_{i}+\beta_{i} p_{i+1}, \alpha_{i}, \beta_{i} \geq 0$. Hence, we can calculate (13) as

$$
\begin{aligned}
v_{i} & =\left\{a_{i}+A_{i}\left(\alpha_{i} p_{i}+\beta_{i} p_{i+1}\right)\right\} \cdot \frac{p_{i}-p_{i+1}}{\left\|p_{i}-p_{i+1}\right\|} \\
& =-\omega_{i} \sqrt{\left(p_{i}^{1}-p_{i+1}^{1}\right)^{2}+\left(p_{i}^{2}-p_{i+1}^{2}\right)^{2}} .
\end{aligned}
$$

Note that $\lambda_{i}$ does not appear in (14). From (14), we can see that the parameters $\alpha_{i}$ and $\beta_{i}$ vanish, and hence $v_{i}$ is constant at any point $x \in D_{i}$. Since $v_{i}$ does not vanish at any point $x \in D_{i}$, the system (2) with (28) does not have any equilibrium points in $M(\varepsilon)$.

Now, a definition on the concept "traversal" for the system (2) with (28) is given as follows [11].

Definition 2 : Let $\Sigma$ be a line segment in the positively invariant, bounded and closed set $M(\varepsilon)$. If the value of an inner product of the unit normal vector to $\Sigma: e_{\Sigma}$ and the velocity vector of the $N$-modal and 2-dimensional piecewise affine system (2) with (28) is not equal to 0 and its sign does not change at any point in $\Sigma$, then $\Sigma$ is said to be traversal with respect to the system (2) with (28).

In addition to Lemma 2 , under the condition of $\omega_{i}>0(i=$ $1, \cdots, N)$, a solution trajectory vector of the system (2) with (28) always has a velocity component in the counterclockwise 
rotation. On the other hand, under the condition of $\omega_{i}<0(i=$ $1, \cdots, N)$, a solution trajectory vector of (2) with (28) always has a velocity component in the clockwise rotation. From this fact, we can derive the following lemma.

Lemma 3 : For the $N$-modal and 2-dimensional piecewise affine system (2) with (28), assume that $\omega_{i}>0(i=1, \cdots, N)$ or $\omega_{i}<0(i=1, \cdots, N)$ holds. Then, there exists a traversal line segment $\Sigma$ at any point in $x \in M(\varepsilon)$, and it is satisfied that $x \in \Sigma$ and $\Sigma$ infinitely intersects with solution trajectories of the system (2) with (28).

(Proof) We assume that $\omega_{i}>0(i=1, \cdots, N)$ or $\omega_{i}<$ $0 \quad(i=1, \cdots, N)$ holds. Then, a solution trajectory of the system (2) with (28) always circles to the counterclockwise rotation or to the clockwise rotation. Now, for a point $x \in M$, we consider a half line whose origin is $O$ and that passes through $x$, and define a subset $\Sigma \subset M$ as the intersection of the half line and $M$. Since the velocity vector field of the system (2) with (28) always has the velocity component of the counterclockwise rotation or the clockwise rotation, the inner product of a normal vector of $\Sigma$ and the vector field of the system (2) with (28) at any point in $\Sigma$ is not equal to 0 and its sign does not change, that is, $\Sigma$ is traversal. Moreover, in each $M_{i}(i=1, \cdots, N)$, since the velocity vector field of the system (2) with (28) always has the velocity component of the clockwise rotation or the counterclockwise rotation, a solution trajectory of the system (2) with (28) $x(t)$ that intersected $\Sigma$ intersects $\Sigma$ in a finite time again. Consequently, it turns out the solution trajectory intersects $\Sigma$ infinitely.

We have to note that the result in Lemma 3 does not depend on $\lambda_{i}(i=1, \cdots, N)$. Using Lemmas $1-3$, we can derive the main theorem on the existence of the limit cycle of the system (2) with (28).

Theorem 2 : For the $N$-modal and 2-dimensional piecewise affine system (2) with (28), assume that $\omega_{i}>0(i=1, \cdots, N)$ or $\omega_{i}<0(i=1, \cdots, N)$ holds. Then, the unique and stable limit cycle of the system (2) with (28) is equivalent to $C$.

(Proof) By the result on the hybrid Poincare-Bendixson theorem derived in [3], [11], it turns out that sufficient conditions for the existence of stable limit cycles of the system (2) with (28) in $M(\varepsilon)$ are the following three: (i) $M(\varepsilon)$ is a positively invariant, bounded and closed set, (ii) there do not exist any equilibrium points at the boundary and in the interior of $M(\varepsilon)$ (iii) there exists a traversal line segment $\Sigma \subset M(\varepsilon)$ such that $x \in \Sigma$ and $\Sigma$ infinitely intersects with solution trajectories of the system (2) with (28). Since we have confirmed these three conditions in Lemma 1, 2, and 3, we can see that there exists a stable limit cycle in $M(\varepsilon)$ for the system (2) with (28) for any admissible $\varepsilon$. Moreover, since $M(\varepsilon)$ converges to $C$ as the values of $\varepsilon$ goes to 0 , it can be confirmed that $C$ is a unique and stable limit cycle. Hence, the proof is completed.

In this paper, we consider an additional parameter $\lambda_{i}$ in the system (2), (28). However, from the results obtained in this subsection, we can see that the existence and the uniqueness of a limit cycle of the system (2), (28) are independent of $\lambda_{i}$. This fact is quite important in the next section.

\section{Theoretical Analysis}

Finally, this subsection gives theoretical analysis on rotational directions and periods of limit cycle solution trajectories of the system (2) with (28). First, we consider the relationship between rotational directions of limit cycles and the parameters in (28). The following proposition can be derived.

Proposition 1 : For the $N$-modal and 2-dimensional piecewise affine system (2) with (28), its limit cycle solution trajectory moves in the counterclockwise rotation for $\omega_{i}>0 \quad(i=$ $1, \cdots, N)$, and conversely it moves in the clockwise rotation for $\omega_{i}<0(i=1, \cdots, N)$.

(Proof) The proof of this proposition is trivial from the discussion in the previous section.

From Proposition 1, it is confirmed that the rotational directions of limit cycles do not depend on $\lambda_{i}$. Next, we analyze periods of limit cycles of the system (2) with (28). It can be expected that after a solution trajectory of the system (2) with (28) converges to $C$, it behaves as a periodic trajectory. By calculating the velocity component of the vector of the system along $C$, we can derive the next proposition.

Proposition 2 : When a limit cycle solution trajectory of the $N$-modal and 2-dimensional piecewise affine system (2) with (28) is sufficiently close to $C$, the period with which it rotates around $C$ is given by

$$
T \approx \sum_{i=1}^{N} \frac{1}{\left|\omega_{i}\right|}
$$

(Proof) The velocity component of a solution trajectory $v_{i}$ of (2) with (28) in $D_{i}$ to the direction of $p_{i}-p_{i+1}$ is given by (13). The length of $C_{i}: L_{i}$ can be calculated as

$$
L_{i}=\sqrt{\left(p_{i}^{1}-p_{i+1}^{1}\right)^{2}+\left(p_{i}^{2}-p_{i+1}^{2}\right)^{2}} .
$$

Therefore, we can obtain the period $T$ as

$$
T \approx \sum_{i=1}^{N} \frac{L_{i}}{\left|v_{i}\right|}=\sum_{i=1}^{N} \frac{1}{\left|\omega_{i}\right|}
$$

This completes the proof of this proposition.

From Proposition 2, we can also see that the period of a limit cycle solution trajectory of the system (2), (28) is not independent of $\lambda_{i}$. So, we can freely choose the value of $\lambda_{i}$.

\section{LIMIT CYCLE CONTROL FOR PIECEWISE AFFINE SYSTEMS}

\section{A. Formulation of Limit Cycle Control}

In this section, we consider a controller design problem on generation of limit cycles for given piecewise affine control systems. First, this sub-section gives the problem formulation. Consider the next piecewise affine control system defined in $D_{i}$ :

$$
\dot{x}=a_{i}+A_{i} x+b_{i} u, \quad x \in D_{i},
$$


where $u \in \mathbf{R}$ is the control input and $b_{i} \in \mathbf{R}^{2}$ is the coefficient vector for the control input. We next consider the state feedback law:

$$
u=k_{i} x+l_{i}, \quad x \in D_{i}
$$

where $k_{i} \in \mathbf{R}^{2}$ and $l_{i} \in \mathbf{R}$. We assume that $p_{i}, p_{i+1}, a_{i}, A_{i}$ are given parameters. Now, we formulate a problem on generating a desired limit cycle for the piecewise affine control system (2) and the state feedback law (19) as follows.

Problem 2 : For the $N$-modal and 2-dimensional piecewise affine control system (18) with the state feedback law (19), design $b_{i}, k_{i}, l_{i}, \omega_{i}, \lambda_{i}(i=1, \cdots, N)$ such that a given polygonal closed curve $C(1)$ is a unique and stable limit cycle of the closed-loop system.

Throughout this paper, we call Problem 2 a limit cycle control problem for piecewise affine control system.

\section{B. Matching Conditions for Limit Cycle Control Problem}

The purpose of this subsection is to derive a solution method of Problem 2 for the piecewise affine control system (18) with the state feedback law (19). To fulfill this, we shall utilize the limit cycle synthesis method obtained in Section 2 . The results in Section II show that the unique and stable limit cycle of the system (2), (28) coincides with $C$. Hence, by tuning design parameters $b_{i}, k_{i}, l_{i}, \omega_{i}, \lambda_{i}(i=1, \cdots, N)$, we conform the closed-loop system (18), (19) to the system (2), (28). We here call the system (2), (28) the reference system. Use the following notations for the system (2), (28):

$$
\begin{aligned}
& a_{i}=\left[\begin{array}{l}
a_{i}^{1} \\
a_{i}^{2}
\end{array}\right], A_{i}=\left[\begin{array}{ll}
A_{i}^{11} & A_{i}^{12} \\
A_{i}^{21} & A_{i}^{22}
\end{array}\right], \\
& b_{i}=\left[\begin{array}{l}
b_{i}^{1} \\
b_{i}^{2}
\end{array}\right], \quad k_{i}=\left[\begin{array}{ll}
k_{i}^{1} & k_{i}^{2}
\end{array}\right] .
\end{aligned}
$$

Conditions such that the closed-loop system (18), (19) is consistent with the reference system (2), (28) can be obtained by the following theorem.

Theorem 3 : The $N$-modal and 2-dimensional piecewise affine control system (18) with the state feedback control law (19) is equivalent to the reference system (2), (28) if and only if the matching conditions:

$$
\begin{aligned}
& a_{i}^{1}+b_{i}^{1} l_{i}=-\lambda_{i}\left(p_{i}^{2}-p_{i+1}^{2}\right)\left(p_{i}^{1} p_{i+1}^{2}-p_{i}^{2} p_{i+1}^{1}\right) \\
& -\omega_{i}\left(p_{i}^{1}-p_{i+1}^{1}\right) \\
& a_{i}^{2}+b_{i}^{2} l_{i}=\lambda_{i}\left(p_{i}^{1}-p_{i+1}^{1}\right)\left(p_{i}^{1} p_{i+1}^{2}-p_{i}^{2} p_{i+1}^{1}\right) \\
& -\omega_{i}\left(p_{i}^{2}-p_{i+1}^{2}\right) \\
& A_{i}^{11}+b_{i}^{1} k_{i}^{1}=-\lambda_{i}\left(p_{i}^{2}-p_{i+1}^{2}\right)^{2} \\
& A_{i}^{12}+b_{i}^{1} k_{i}^{2}=\lambda_{i}\left(p_{i}^{2}-p_{i+1}^{2}\right)\left(p_{i}^{1}-p_{i+1}^{1}\right) \\
& A_{i}^{21}+b_{i}^{2} k_{i}^{1}=\lambda_{i}\left(p_{i}^{2}-p_{i+1}^{2}\right)\left(p_{i}^{1}-p_{i+1}^{1}\right) \\
& A_{i}^{22}+b_{i}^{2} k_{i}^{2}=-\lambda_{i}\left(p_{i}^{1}-p_{i+1}^{1}\right)^{2}
\end{aligned}
$$

hold.

(Proof) Substituting (19) into (18), we get the closed-loop system:

$$
\begin{aligned}
\dot{x} & =a_{i}+A_{i} x+b_{i}\left(k_{i} x+l_{i}\right) \\
& =a_{i}+b_{i} l_{i}+\left(A_{i}+b_{i} k_{i}\right) x \\
& =\left[\begin{array}{c}
a_{i}^{1}+b_{i}^{1} l_{i} \\
a_{i}^{2}+b_{i}^{2} l_{i}
\end{array}\right]+\left[\begin{array}{cc}
A_{i}^{11}+b_{i}^{1} k_{i}^{1} & A_{i}^{12}+b_{i}^{1} k_{i}^{2} \\
A_{i}^{21}+b_{i}^{2} k_{i}^{1} & A_{i}^{22}+b_{i}^{2} k_{i}^{2}
\end{array}\right] x .
\end{aligned}
$$

Comparing the components of the reference system (2), (28) to the closed-loop system (27), we can obtain the matching conditions (21)-(26).

The matching conditions (21)-(26) consists of 6 algebraic equations, and 7 unknown variables: $b_{i}^{1}, b_{i}^{2}, k_{i}^{1}, k_{i}^{2}, l_{i}, \omega_{i}, \lambda_{i}$. Hence, by solving them under the condition $\lambda_{i}>0$, we can obtain these unknown variables, that is, a solution of Problem 2 .

\section{SIMULATIONS}

This section presents a numerical example in order to confirm the effectiveness of the results derived in the previous sections. We now give data of a polygon with $N=4$ as $P_{1}=(1,0), P_{2}=(0,1), P_{3}=(-1,0), P_{4}=(0,-1) .0$ The polygon is shown in Fig. 4.

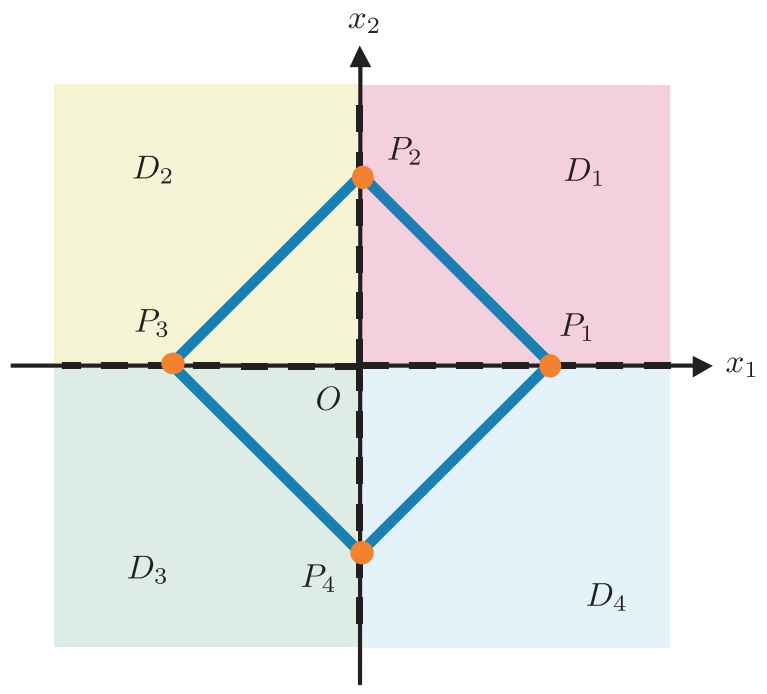

Fig. 4 : Polygonal Closed Curve of Example by

The coefficients of the piecewise affine system are given

$$
\begin{aligned}
& a_{1}=\left[\begin{array}{c}
-3 \\
2
\end{array}\right], A_{1}=\left[\begin{array}{ll}
0 & 1 \\
1 & 3
\end{array}\right], \\
& a_{2}=\left[\begin{array}{l}
-1 \\
-3
\end{array}\right], A_{2}=\left[\begin{array}{cc}
-1 & 0 \\
0 & 2
\end{array}\right], \\
& a_{3}=\left[\begin{array}{c}
1 \\
-1
\end{array}\right], A_{3}=\left[\begin{array}{cc}
-2 & 1 \\
1 & 1
\end{array}\right], \\
& a_{4}=\left[\begin{array}{l}
9 \\
1
\end{array}\right], A_{4}=\left[\begin{array}{cc}
-8 & 0 \\
0 & -8
\end{array}\right] .
\end{aligned}
$$

By solving the matching conditions for Problem 2 (21)(26) in terms of the problem formulation, we can calculate 
design parameters as follows:

$$
\begin{aligned}
& b_{1}=\left[\begin{array}{l}
1 \\
2
\end{array}\right], k_{1}=\left[\begin{array}{ll}
-1 & -2
\end{array}\right], \\
& l_{1}=1, \omega_{1}=3, \lambda_{1}=1, \\
& b_{2}=\left[\begin{array}{c}
-1 \\
2
\end{array}\right], k_{2}=\left[\begin{array}{ll}
1 & -2
\end{array}\right], \\
& l_{2}=2, \omega_{2}=1, \lambda_{2}=2, \\
& b_{3}=\left[\begin{array}{c}
-1 \\
2
\end{array}\right], \quad k_{3}=\left[\begin{array}{ll}
1 & -2
\end{array}\right], \\
& l_{3}=2, \omega_{3}=2, \lambda_{3}=3, \\
& b_{4}=\left[\begin{array}{l}
1 \\
1
\end{array}\right], \quad k_{4}=\left[\begin{array}{ll}
4 & 4
\end{array}\right], \\
& l_{4}=-4, \omega_{4}=1, \lambda_{4}=4 .
\end{aligned}
$$

Note that $\lambda_{i}>0 \quad(i=1,2,3,4)$ holds in (29). It can be confirmed that from Proposition 1, a limit cycle solution trajectory moves in the counterclockwise rotation since $\omega_{i}>$ $0(i=1,2,3,4)$ holds. In addition, from Proposition 2 we can estimate the period of a limit cycle solution trajectory as

$$
T \approx \sum_{i=1}^{4} \frac{1}{\left|\omega_{i}\right|}=\frac{17}{6}
$$

We set the initial state as $x_{0}=[1,1]^{\top}$ for the numerical simulation. The simulation results are illustrated in Figs. 5-7. Fig. 5 shows the solution trajectory on the $x_{1} x_{2}$-plane. In Figs. 6 and 7, the time series of $x_{1}$ and $x_{2}$ are shown, respectively. From these simulation results, we can see that the solution trajectory that starts from $x_{0}$ behaves as a limit cycle for the desired polygonal closed curve $C$, and hence Theorem 1 holds. As we expected above, the solution trajectory moves in the counterclockwise rotation, and this result is coincident with Proposition 1. Moreover, the estimated period $T \approx 17 / 6$ is mostly agree about the simulation result from Figs. 6 and 7.

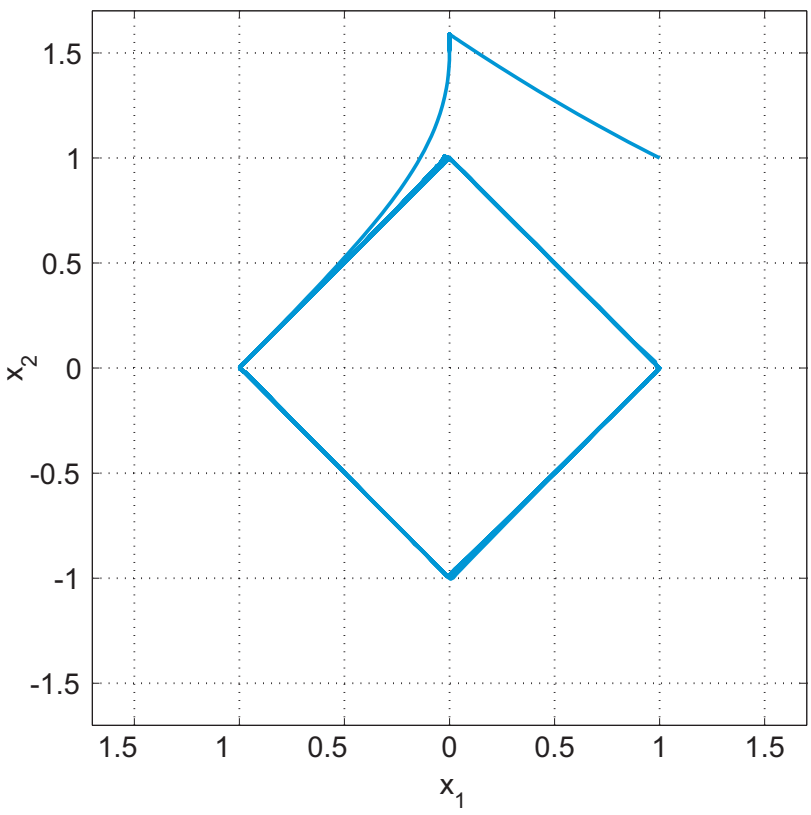

Fig. 5 : Solution Trajectory on $x_{1} x_{2}$-Plane

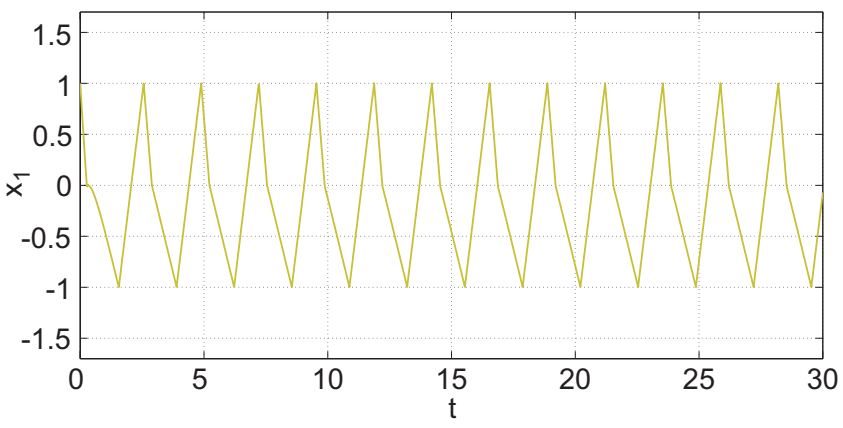

Fig. 6 : Time Series of $x_{1}$

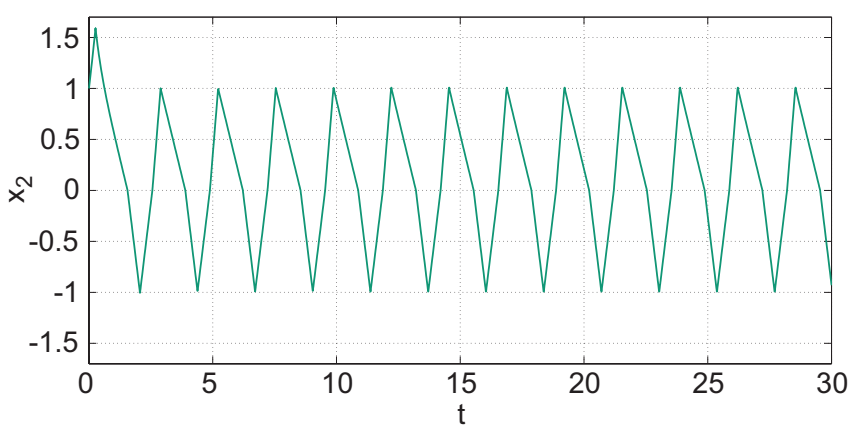

Fig. 7 : Time Series of $x_{2}$

\section{CONCLUSION}

In this paper, we have considered a limit cycle control problem for a multi-modal and 2-dimensional piecewise control affine system. We have derive the matching conditions such that the piecewise control affine system with the state feedback law corresponds with the reference system which generates a unique and stable limit cycle. It has been confirmed by solving the matching conditions we can obtain the values of design parameters. A numerical simulations show the availability and the application potentiality of the proposed method.

Our future work includes applications of the proposed control method to real systems and extensions to multidimensional piecewise affine systems.

\section{REFERENCES}

[1] J. Buchli, L. Righetti and A. Ijspeert, "Engineering Entrainment and Adaptation in Limit Cycle Systems," Biological Cybernetics, vol.95, pp.645-664, 2006

[2] D. N. Green, "Synthesis of Systems with Periodic Solutions Satisfying $\mathcal{V}(x)=0$," IEEE Trans. Circuits and Systems, vol.31, no.4, pp.317326, 1984

[3] S. N. Simic, K. H. Johansson, J. Lygeros and S. Sastry, "Hybrid Limit Cycles and Hybrid Poincare-Bendixson," in Proc. of IFAC World Congress, Barcelona, Spain, pp.86-89, 2002

[4] A. Girard, "Computation and Stability Analysis of Limit Cycles in Piecewise Linear Hybrid Systems," in Proc. of 1st IFAC Conference on Analysis and Design of Hybrid Systems, Saint-Malo, France, pp. 181-186, 2002

[5] M. Adachi and T. Ushio, "Synthesis of Hybrid Systems with Limit Cycles Satisfying Piecewise Smooth Constant Equations," IEICE Trans. Fundamentals, vol.E87-A, No.4, pp.837-842, 2004

[6] F. Gómez-Estern, J. Aracil, F. Gordillo and A. Barreiro, "Generation of Autonomous Oscillations via Output Feedback," in Proc. of IEEE CDC 2005, Seville, Spain, pp.7708-7713, 2005 
[7] A. Ohno, T. Ushio and M. Adachi, "Synthesis of Nonautonomous Systems with Specified Limit Cycles," IEICE Trans. Fundamentals, vol.E89-A, No.10, pp.2833-2836, 2006

[8] F. Gómez-Estern, A. Barreiro, J. Aracil and F. Gordillo, "Robust Generation of Almost-periodic Oscillations in a Class of Nonlinear systems," Int. J. Robust Nonlinear Control, vol.16, no.18, pp.863-890, 2006

[9] D. Flieller, P. Riedinger, and J. Louis, "Computation and Stability of Limit Cycles in Hybrid Systems," Nonlinear Analysis, Vol. 64, pp. 352367, 2006

[10] T. Kai and R. Masuda, "Limit Cycle Synthesis of Multi-Modal and 2Dimensional Piecewise Affine Systems," Mathematical and Computer Modelling, Vol. 55, pp. 505-516, 2012

[11] M. Suenaga and T. Hayakawa, "Existence Condition of Periodic Orbits for Piecewise Affine Planar Systems," in Proc. of SICE 8th Annual Conference on Control Systems, Kyoto, Japan, 2008

[12] T. Kai and R. Masuda, "Controller Design for 2-Dimensional Nonlinear Control Systems Generating Limit Cycles and Its Application to Spacerobots," in Proc. of NOLTA 2008, Budapest, Hungary, pp.496499, 2008

[13] T. Kai and M. Katsuta, "Limit Cycle Control for 2-Dimensional Discrete-time Nonlinear Control Systems and Its Application to Chaos Systems," in Proc. of NOLTA 2009, Sapporo, Japan, pp.86-89, 2009

[14] A. Schild, Xu Chu Ding, M. Egerstedt, J. Lunze, "Design of optimal switching surfaces for switched autonomous systems," in Proc. of IEEE Conference on Decision and Control \& Chinese Control Conference 2009, Shanghai, China, pp. 5293-5298, 2009

[15] T. Kai and R. Masuda, "A Limit Cycle Synthesis Method of MultiModal and 2-Dimensional Piecewise Affine Systems," in Proc. of 50th IEEE Conference on Decision and Control and European Control Conference, Orlando, USA, pp. 4759-4764, 2011 